\title{
Effectiveness of a 5-Day External Stenting Protocol on Urological Complications After Renal Transplantation
}

\author{
Robert C. Minnee · Frederike J. Bemelman • \\ Pilar P. Laguna Pes · Ineke J. M. ten Berge • \\ Dink A. Legemate · Mirza M. Idu
}

Published online: 23 September 2009

(c) The Author(s) 2009. This article is published with open access at Springerlink.com

\begin{abstract}
Background Ureteral stents are successful in reducing urological complications after renal transplantation. However, the optimal duration and method of stenting have not yet been clarified. The objective of the present study was to investigate the frequency of urological complications when a 5-day external stented ureterocystostomy protocol was followed.

Methods A single-center nonrandomized analysis of 392 kidney transplantations between June 2003 and June 2007 was conducted. From July 2005 all 196 renal transplant recipients received a 5-day external stented ureterocystostomy. A urological complication was defined as any cause leading to the placement of a percutaneous nephrostomy catheter and/or surgical revision of the ureterocystostomy. Results In the non-stented group, 21 of the 196 patients (10.7\%) developed a urological complication compared to 13 patients $(6.6 \%)$ in the stented group $(p=0.151)$. In the stented group, 2 of the 66 recipients of a living donor transplant $(3.0 \%)$ developed a urological complication compared to 8 of the 59 recipients (13.6\%) in the non-stented
\end{abstract}

R. C. Minnee · D. A. Legemate · M. M. Idu ( $ه)$ Department of Surgery, Academic Medical Center, Meibergdreef 9, Amsterdam, AZ 1105, The Netherlands e-mail: m.m.idu@amc.uva.nl

R. C. Minnee

e-mail: rcminnee@hotmail.com

F. J. Bemelman · I. J. M. ten Berge

Renal Transplant Unit, Department of Nephrology,

Academic Medical Center, Meibergdreef 9,

Amsterdam, AZ 1105, The Netherlands

P. P. Laguna Pes

Department of Urology, Academic Medical Center,

Meibergdreef 9, Amsterdam, AZ 1105, The Netherlands group $(P=0.030)$. Eleven of 130 recipients of a deceased donor transplant $(8.5 \%)$ in the stented group developed a urological complication, compared to 13 of the 137 recipients $(9.5 \%)$ in the non-stented group $(P=0.769)$. The surgical revision rate of the stented and the non-stented group was $5 / 1339 \%$ and $6 / 2129 \%$, respectively.

Conclusions A 5-day routine external stent protocol is efficacious in living donor renal transplantation in preventing early postoperative ureter obstruction, but this stenting period seems inadequate for deceased donor renal transplantation.

\section{Introduction}

Urological complications remain an important source of morbidity and occasionally mortality, after renal transplantation. The two major urological complications after renal transplantation are urinary leakage and obstruction, mostly located at the ureterovesical junction or in the distal transplant ureter.

Two meta-analyses have demonstrated that on comparison with a non-stented ureterocystostomy, a stented ureterocystostomy leads to a significantly lower urological complication rate (odds ratio $0.24,95 \%$ confidence interval [CI] $0.07-0.77 ; P=0.02)[1,2]$. This finding has been confirmed by two recent studies, one of which showed that stenting was more cost-effective. Accordingly, we changed our surgical technique from non-stented to stented ureterocystostomy $[3,4]$.

However, using a routine stenting protocol, the number needed to treat (NNT) to prevent one urological complication is high, ranging from 10 to 30 [2]. In addition, the optimal duration of stenting and method of stenting have yet to be determined. 
The aim of the present study was to analyze the results of a short (5-day) external stented ureterocystostomy protocol on the occurrence of urological complications.

\section{Patients and methods}

All 392 consecutive patients who underwent renal transplantation between June 2003 and June 2007 at the Academic Medical Center, Amsterdam, were included in the study. From July 2005 all 196 renal transplant recipients received a 5-day external stented ureterocystostomy. All procedures were single renal transplants performed through an extraperitoneal approach in the iliac fossa. The renal vein was anastomosed to the external iliac vein, and the renal artery was anastomosed to the external iliac artery. The method used to establish urinary continuity was either the extravesical ureterocystostomy (Lich-Gregoir method) or the intravesical ureterocystostomy (Politano-Leadbetter method), according to the personal preference of the surgeon. The ureteroneocystostomy was stented with an externally draining 8 French (Fr) catheter for 5 days. The stent was introduced into the bladder through a direct suprapubic bladder puncture and positioned in the transplant renal pelvis. The stent drained externally and was sutured to the bladder mucosa and to the skin. Postoperatively all patients had an indwelling bladder catheter. The operation day was counted as day 0 . The stent was routinely removed on the fifth postoperative day.

The bladder catheter was removed in all patients on day 7 after urinary leakage had been excluded by cystography on the same day. All patients were followed at our center for at least 1 year after successful transplantation. After 1 year, patients were transferred to their referral center. Standard immunosuppression consisted of prednisolone, a calcineurin inhibitor, mycophenolate mofetil, and prophylactic antiCD25 monoclonal antibody (basiliximab). Initial episodes of acute rejection were treated with pulse doses of methylprednisolone; second episodes, with thymoglobulin. Delayed graft function was defined as the need for dialysis within the first postoperative week. Renal transplant function was monitored by serial serum and urine creatinine, urinary output, and renography. Renal graft failure was defined as removal of the graft or loss of function requiring return to dialysis. After transplantation, the urinary output volumes through the stent and the indwelling bladder catheter were collected separately each day. These urinary output volumes were only measured in the stented group. A urological complication was defined as any cause (e.g., urinary fistula, leakage, ureteral obstruction) requiring a percutaneous nephrostomy catheter and/or surgical revision. Urinary tract infections and vesicoureteral reflux were not counted as urological complications. Urinary tract infection was defined as bacteriuria confirmed by a positive urine culture. If indicated, a percutaneous nephrostomy catheter was inserted and antegrade pyelography was performed. The nephrostomy catheter was left in place to maintain renal excretory function. Routinely, the nephrostomy catheter was changed every 6 weeks at our outpatient clinic. If the urinary obstruction persisted despite a well-functioning percutaneous nephrostomy catheter, an operative reconstruction was usually performed 3-6 months later.

\section{Statistical analysis}

Comparisons of categorical data were performed using the Chi-square test. Continuous data were compared between the groups using the Mann-Whitney $U$-test. Univariate logistic regression analysis was performed to identify risk factors for urological complication. The graft survival rates were calculated by the Kaplan-Meier technique and the log-rank test. A $P$ value of $<0.05$ was considered statistically significant. For statistical analyses the SPSS software package (SPSS 14.0.2, Chicago, IL) was used.

\section{Results}

Donor and recipient characteristics are presented in Table 1. Of the 392 consecutive patients receiving renal transplants, 34 developed a urological complication (8.7\%). In the nonstented group 21 of the first 196 patients (10.7\%) developed a urological complication, compared to 13 patients of the 196 patients $(6.6 \%)$ in the stented group $P=0.151$ (Table 2). Two patients in the stented group did not receive a ureter stent, because of a very small bladder. One of these patients developed a urological complication. In the stented group, 2 of the 66 recipients of a living donor transplant $(3.0 \%)$ developed a urological complication compared to 8 of the 59 recipients $(13.6 \%)$ in the non-stented group $P=0.030$. Eleven of 130 recipients of a deceased donor transplant $(8.5 \%)$ in the stented group developed a urological complication, compared to 13 of the 137 recipients $(9.5 \%)$ in the non-stented group $P=0.769$. In the stented group, the 13 urological complications included 10 ureteral strictures (76.9\%) and 3 ureteral leaks. In 8 of these 13 patients, the urological complication was managed using a temporary percutaneous nephrostomy catheter only. In the remaining 5, surgical revision (a neo-ureterocystostomy) was necessary to correct the urological complication. In the non-stented group, the 21 urological complications included 15 ureteral strictures $(71.4 \%)$ and 6 ureteral leaks. In 15 of these 21 patients, the urological complication was managed using a temporary percutaneous nephrostomy catheter only. The remaining six patients underwent a surgical revision. In both 
Table 1 Demographics of donors and recipients

\begin{tabular}{lccc}
\hline Variable & $\begin{array}{c}\text { No stent } \\
(n=196)\end{array}$ & $\begin{array}{l}\text { Stent } \\
(n=196)\end{array}$ & $P$ value \\
\hline $\begin{array}{l}\text { Donor } \\
\text { Male }\end{array}$ & $80(41 \%)$ & $80(41 \%)$ & 0.452 \\
Age & & & \\
$\quad<18$ years & $14(7 \%)$ & $10(5 \%)$ & 0.188 \\
18-40 years & $49(25 \%)$ & $65(33 \%)$ & 0.145 \\
$40-65$ years & $120(61 \%)$ & $110(56 \%)$ & 0.537 \\
$>65$ years & $13(7 \%)$ & $11(6 \%)$ & 0.836 \\
Left kidney & $81(41 \%)$ & $113(58 \%)$ & 0.005 \\
Living related transplantation & $59(30 \%)$ & $66(34 \%)$ & 0.448 \\
Recipient & & & \\
Male & $121(62 \%)$ & $101(52 \%)$ & 0.047 \\
Age & & & \\
$\quad<18$ years & $13(6 \%)$ & $13(7 \%)$ & 0.695 \\
18-40 years & $54(28 \%)$ & $50(25 \%)$ & 0.647 \\
40-65 years & $108(55 \%)$ & $115(59 \%)$ & 0.475 \\
$>65$ years & $19(9 \%)$ & $18(9 \%)$ & 0.863 \\
First transplantation & $164(84 \%)$ & $163(83 \%)$ & 0.892 \\
Second transplantation & $29(15 \%)$ & $24(12 \%)$ & 0.460 \\
Third and fourth transplantations & $3(1 \%)$ & $9(5 \%)$ & 0.079 \\
\hline
\end{tabular}

groups, no recurrent urological complication occurred after operation, and no reoperations were necessary. In both groups, the majority of complications occurred within the first postoperative month (Fig. 1). After placement, the percutaneous nephrostomy catheter remained in situ for varying lengths of time. The mean $( \pm \mathrm{SD})$ percutaneous nephrostomy catheter time (days) was $146 \pm 40$ (range: $12-$ 411 days) in the stented group and $155 \pm 45$ days (range: $17-440$ days) in the non-stented group. Urinary tract infection in the stented group occurred in 41 patients (20.9\%), compared to 45 patients (22.9\%) in the non-stented group $P=0.689$. The surgical outcomes are shown in Table 3 .

During the first 5 days, the urinary output volumes via the stent were significantly lower in the urological complication group than in the non-urological complication group ( $P=0.026)$ (Fig. 1). During this period no differences in urinary output volume through the indwelling bladder catheter between these groups were found $(P=0.278)$.

The 1-month survival in the two groups was identical at $93 \%$ and that at 1 year the survival rate was again identical at $89 \%$.

\section{Discussion}

A routine short-duration stenting protocol of 5 days resulted in a urological complication rate of $6.6 \%$ for all
Table 2 Urological complications and treatment

\begin{tabular}{lcrl}
\hline & No stent $(n=196)$ & Stent $(n=196)$ & $P$ value \\
\hline \multicolumn{4}{l}{ Urological complications } \\
Living donor & $8 / 59(13.6) \%$ & $2 / 66(3.0) \%$ & 0.030 \\
Deceased donor & $13 / 137(9.5 \%)$ & $11 / 130(8.5 \%)$ & 0.769 \\
Surgical revision & $6 / 21(29) \%$ & $5 / 13(39) \%$ & 0.760 \\
\hline
\end{tabular}

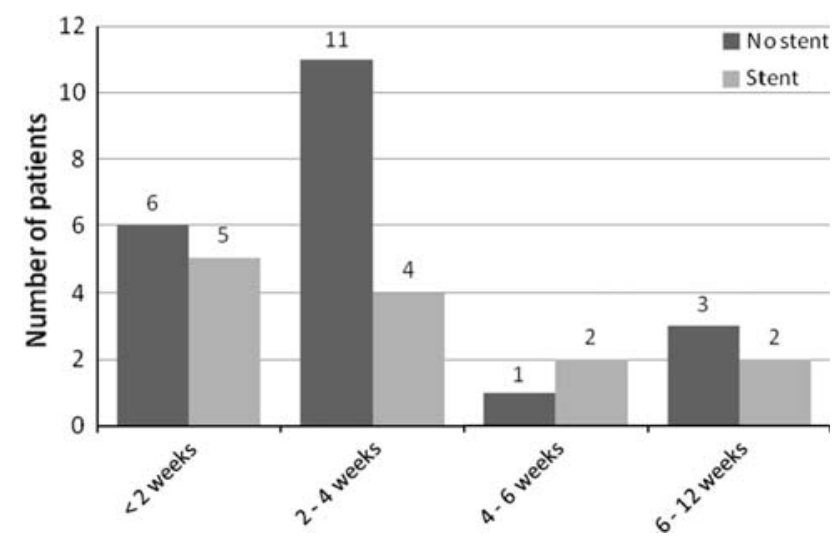

Fig. 1 Time interval between the operation and occurrence of the urological complication

renal transplants. There was a urological complication rate of $3.0 \%$ in transplants from living donors and $8.5 \%$ from deceased donors. Compared with our non-stented ureteroneocystostomy group, the overall urological complication rate at our center was reduced from 10.7 to $6.6 \%$. In the living donor group, the urological complication rate was reduced from 13.6 to $3.0 \%$. In the deceased donor group it was reduced from 9.5 to $8.5 \%$.

Classically, the two major etiological factors for urological complications after renal transplantation are surgical-technical factors and distal transplant ureteral ischemia. Surgical-technical factors include poor harvesting and ureterocystostomy techniques. Measures including the preservation of the periureteral vessels and fat, avoidance of large incisions in the bladder, the reduction of ureteral length, avoidance of external ureteral compression by the vas deferens, and creating a watertight urinary anastomosis, all decrease the incidence of urological complications [5]. The most frequent causes of urinary leakage are necrosis and suture failure [6], whereas ureteral strictures might result from intraluminary factors, such as calculi, blood clots, or extraluminary factors such as compression of blood and lymphatic fluid [5].

A ureterocystostomy protocol in a selected group of transplant recipients would be an option to reduce the high NNT for routine stenting. However, to date no useful preoperative and/or perioperative factors have been identified that would serve to predict postoperative urological 
Table 3 Surgical outcomes in all recipients

${ }^{a}$ Values in parentheses are $95 \%$ confidence intervals

\begin{tabular}{lcclc}
\hline & $\begin{array}{l}\text { No stent } \\
(n=196)\end{array}$ & $\begin{array}{l}\text { Stent } \\
(n=196)\end{array}$ & & Univariate analysis \\
\cline { 5 - 5 } & & & Odds ratio $^{\mathrm{a}}$ & $P$ value \\
\hline Left fossa iliaca & $56(29)$ & $46(24)$ & $1.30(0.83-2.05)$ & 0.250 \\
Cold ischemia time $>24 \mathrm{~h}$ & $28(14)$ & $25(13)$ & $1.14(0.64-2.04)$ & 0.658 \\
Anastomosis time $>45 \mathrm{~min}$ & $22(11)$ & $20(10)$ & $1.11(0.59-2.11)$ & 0.744 \\
Arterial reconstruction & $29(15)$ & $27(14)$ & $1.09(0.62-1.92)$ & 0.773 \\
Vein reconstruction & $27(14)$ & $18(9)$ & $1.58(0.84-2.97$ & 0.154 \\
Extravesical uterocystostomy & $185(94)$ & $183(93)$ & $1.20(0.52-2.74)$ & 0.673 \\
Urological complication & $21(11)$ & $13(7)$ & $1.69(0.82-3.48)$ & 0.151 \\
Rejection & $54(28)$ & $53(27)$ & $1.03(0.66-1.60)$ & 0.910 \\
Delayed graft function & $62(32)$ & $60(31)$ & $0.98(0.64-1.49)$ & 0.910 \\
\hline
\end{tabular}

complications and can be used for the implementation of a selective stenting protocol [7, 8]. In addition, in a retrospective analysis, Georgiev et al. [8] recently demonstrated that routine stenting of the ureterocystostomy is superior to stenting on demand.

Six of the seven randomized trials that compared stenting with no stenting, used a double-J stent with a treatment duration of between 2 weeks and 3 months [9-14]. The seventh study used an external 8 Fr straight stent for 7-10 days [15]. Side effects and complications of stents, especially the double-J stent, include patient discomfort and irritated bladder symptoms, bacteriuria with or without clinical signs of infection, urosepsis, hematuria, flank and loin pain on the same side as the stent, dislocation of the stent, fragmentation, and calcification [16]. The presence of the double-J stent for a long period increases the risk of urinary tract infection [4]. An additional disadvantage of a double-J stent is the need for a cystoscopy and anesthesia for stent removal. Urological complication rates of double-J stents vary between 0 and 4\% [2]. Junjie et al. [17] examined the duration of stent placement and reported a lower urological complication rate in the group who had a stent for 5-7 days (4.3\%) compared with 3-4 weeks (7.7\%).

Our protocol involved the use of an externally draining 8 Fr catheter for 5 days. An advantage of using this type of stent is that it avoids the complications associated with the double-J stent and the additional cystoscopy required for stent removal. Our urological complication rate from using a double-J stent in the living donor transplantation group is comparable with that found in the literature [1, 2]. Thus, our protocol has the additional advantage of eliminating the extra intervention necessary for stent removal with a comparable complication rate in living donor transplant recipients. However, the 5-day period of stenting in the patients with transplants from deceased donors resulted in a rather high urological complication rate of $8.5 \%$, if compared with a complication rate of less than $2-3 \%$ as reported from several randomized trials [2].
The use of a stent resulted in a significantly increased risk of a urinary tract infection when compared with the non-stented ureterocystostomy (relative risk 1.49, 95\% CI: $1.04-2.15 ; P=0.03$ ) [2]. Our rate was $20.9 \%$, whereas the reported urinary tract infection prevalence was $25.8 \%$ [2]. Indwelling ureteral stents are often associated with the development of recurrent urinary tract infection. Possibly our urinary tract infection rate is lower because of the short duration of stent placement.

The main limitation of the present study is the use of a historical control group instead of a randomized contemporaneous control group.

We conclude that there was significant benefit of stenting in the living donor group. A strong advantage of our short-duration external stenting protocol was not seen in the deceased group, whereas there was a suggestion of trend toward better outcomes with stenting in the patients overall. For future research large randomized controlled studies would be useful, especially in deceased donor kidney transplants.

Open Access This article is distributed under the terms of the Creative Commons Attribution Noncommercial License which permits any noncommercial use, distribution, and reproduction in any medium, provided the original author(s) and source are credited.

\section{References}

1. Mangus RS, Haag BW (2004) Stented versus nonstented extravesical ureteroneocystostomy in renal transplantation: a metaanalysis. Am J Transplant 4:1889-1896

2. Wilson CH, Bhatti AA, Rix DA et al (2005) Routine intraoperative ureteric stenting for kidney transplant recipients. Cochrane Database Syst Rev 2005;(4)CD004925

3. DuBay DA, Lynch R, Cohn J et al (2007) Is routine ureteral stenting cost-effective in renal transplantation? J Urol 178:25092513

4. Tavakoli A, Surange RS, Pearson RC et al (2007) Impact of stents on urological complications and health care expenditure in renal transplant recipients: results of a prospective, randomized clinical trial. J Urol 177:2260-2264 
5. Blanchet P, Hammoudi Y, Eschwege P et al (2000) Urinary complications after kidney transplantation can be reduced. Transplant Proc 32:2769

6. Salvatierra O Jr, Kountz SL, Belzer FO (1974) Prevention of ureteral fistula after renal transplantation. J Urol 112:445-448

7. Minnee RC, Surachno S, Kox C et al (2006) Is a selective splinted ureterocystostomy protocol feasible in renal transplantation? An analysis of 475 renal transplantations. Transpl Int 19:558-562

8. Georgiev P, Boni C, Dahm F et al (2007) Routine stenting reduces urologic complications as compared with stenting "on demand" in adult kidney transplantation. Urology 70:893-897

9. Bassiri A, Amiransari B, Yazdani M et al (1995) Renal transplantation using ureteral stents. Transplant Proc 27:2593-2594

10. Benoit G, Blanchet P, Eschwege $P$ et al (1996) Insertion of a double pigtail ureteral stent for the prevention of urological complications in renal transplantation: a prospective randomized study. J Urol 156:881-884

11. Guleria S, Agarwal S, Kumar R et al (2007) The double J stent: its impact on the urological complications in live-related transplantation. Indian J Urol 14:101-104
12. Kumar A, Kumar R, Bhandari M (1998) Significance of routine JJ stenting in living related renal transplantation: a prospective randomised study. Transplant Proc 30:2995-2997

13. Osman Y, li-El-Dein B, Shokeir AA et al (2005) Routine insertion of ureteral stent in live-donor renal transplantation: is it worthwhile? Urology 65:867-871

14. Pleass HC, Clark KR, Rigg KM et al (1995) Urologic complications after renal transplantation: a prospective randomized trial comparing different techniques of ureteric anastomosis and the use of prophylactic ureteric stents. Transplant Proc 27:1091-1092

15. Dominguez J, Clase CM, Mahalati K et al (2000) Is routine ureteric stenting needed in kidney transplantation? A randomized trial. Transplantation 70:597-601

16. Richter S, Ringel A, Shalev M et al (2000) The indwelling ureteric stent: a 'friendly' procedure with unfriendly high morbidity. BJU Int 85:408-411

17. Junjie M, Jian X, Lixin Y et al (1998) Urological complications and effects of double-J catheter in ureterovesical anastomosis after cadaveric kidney transplantation. Transplant Proc 30:30133014 